\title{
Etude expérimentale de l'écoulement bidimensionnel autour d'un obstacle rectangulaire et un obstacle rectangulaire arrondi
}

\author{
Khaled Aliane $^{1 *}$, Omar Sebbane $^{1}$ et Abderrahim Houmat ${ }^{2}$
}

Département de mécanique, Faculté des sciences de l'ingénieur, Université de Tlemcen, B.P. 230, Tlemcen 13000, Algérie

'Laboratoire des matériaux et énergies renouvelables

${ }^{2}$ Laboratoire de structure et modélisation

(Reçu le 21 Février 2006, accepté le 05 Août 2006)

*Correspondance,courriel : kh_aliane@yahoo.fr

\section{Résumé}

L'étude de l'écoulement bidimensionnel autour d'obstacles en fonction du nombre de Reynolds $R e_{h}$ et du rapport sans dimension $h / H$ ( hauteur de l'obstacle/Hauteur du canal), est envisagée afin d'examiner l'écoulement autour de deux modèles d' obstacles, un obstacle rectangulaire et un obstacle rectangulaire à bout arrondi. Deux régions importantes en aval de l'obstacle sont à étudier : la première c'est la zone de recirculation générée en aval de l'obstacle où on étudie les dimensions des longueurs de réattachement $X_{R} / h$. La deuxième région est la deuxième zone de recirculation.

L'influence de la forme arrondie sur les dimensions des longueurs de réattachement et sur la génération de la deuxième zone de recirculation est illustrée et cela, en comparant les résultats obtenus pour les deux modèles d'obstacles. Les mesures sont effectuées en utilisant l'anémomètre Laser à effet Doppler.

Mots-clés : écoulement, obstacle rectangulaire, obstacle rectangulaire arrondi, longueur de réattachement, deuxième zone de recirculation, anémomètre laser à effet Doppler.

\section{Abstract}

Experimental study of the two-dimensional flow around rectangular block obstacle and a rounded end rectangular obstacle

A study of the two dimensional flow around obstacles in terms of the Reynolds $R e_{h}$ number and the non dimensional ratios $h / H$ (obstacle height to channel height) is considered to examine two models of obstacles, rectangular block obstacle and a 
rounded rectangular obstacle. Two important regions in the downstream side of the obstacle must be studied: the first is the recirculation zone generated in the downstream of the obstacle where we study the values of the reattachment length. The second region is the secondary recirculation zone. The measures are accomplished using LaserDoppler-anemometer. The influence of the rounded form on the reattachment length and on the generation of the secondary recirculation zone is illustrated and this in comparing the results of this model with those of the rectangular obstacle.

Keywords : flow, rectangular block obstacle, a rounded rectangular obstacle, reattachment length, secondary recirculation zone, Laser Doppler Anemometer.

\section{Nomenclature}

$\vec{F} \quad$ Force par unité de surface

$\left(\mathrm{N} / \mathrm{m}^{2}\right)$

$H$ Hauteur du canal

h Hauteur de l'obstacle

I Longueur de l'obstacle

$p$ Pression

$\operatorname{Re}_{h} \quad \mathrm{U}_{\mathrm{g}} \mathrm{h} / \mathrm{V}$ nombre de Reynolds

$U_{g} \quad$ Vitesse moyenne au dessus de l'obstacle

$u \quad$ Vitesse locale suivant la direction $\mathrm{x}$

$v \quad$ Vitesse locale suivant la direction y

$\vec{V} \quad$ Vecteur vitesse

W Largeur de l'obstacle

$x \quad$ coordonnée cartésienne

$X_{R} \quad$ Longueur de réattachement

$X_{1}, X_{2} \quad$ Position des points de séparation et de réattachement respectivement de la deuxième zone de recirculation

y coordonnée cartésienne

$\delta \quad$ Epaisseur de la couche limite

$\mu \quad$ Viscosité dynamique

$v \quad$ Viscosité cinématique

$\rho \quad$ Masse volumique 


\section{Introduction}

Les écoulements de fluides en présence d'obstacles sont largement utilisés dans l'industrie, et leurs applications sont extrêmement variées. On peut les rencontrer dans le cas des problèmes d'environnement liés à la dispersion des polluants à travers les agglomérations ainsi que les effets du vent sur les bâtiments, la ventilation des tunnels routiers, les ailettes de refroidissement des engins thermiques [1], les chicanes des échangeurs de chaleur ou des capteurs solaires, les canalisations urbaines, etc.

L'étude de l'écoulement autour d'obstacle peut se faire suivant deux approches: l'approche numérique où on simule l'écoulement en s'appuyant sur un modèle mathématique; et l'approche expérimentale où on doit acquérir des mesures sur banc d'essai.

- L'approche numérique consiste à discrétiser l'équation gouvernante de l'écoulement par une méthode numérique en utilisant différentes formulations [2,3].

- L'approche expérimentale peut être traitée suivant deux aspects, l'aspect qualitatif où on utilise les techniques de visualisation pour analyser les différentes structures tourbillonnaires naissantes au sein de l'écoulement [4]. Le deuxième aspect est l'aspect quantitatif où les zones de recirculation en aval de l'obstacle sont quantifiées en fonction des paramètres dynamiques de l'écoulement tels que le nombre de Reynolds [5].

L'apport de cette étude consiste à pratiquer un arrondissement sur le bout de l'obstacle rectangulaire et étudier l'influence de cet arrondissement sur les dimensions de la longueur de réattachement $X_{R} / h$ et sur la deuxième zone de recirculation créée en aval de cet obstacle et cela en comparant les résultas obtenus avec ceux de l'obstacle rectangulaire. Les deux modèles d'obstacles étudiés sont illustrés dans la Figure 1.

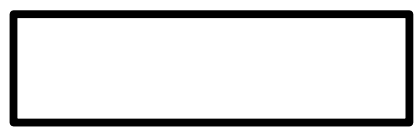

(a)

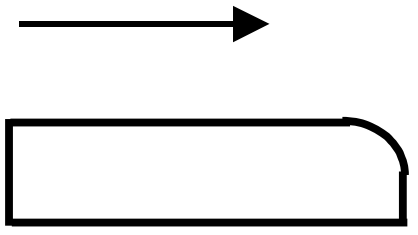

(b)

Figure 1 : Modèles d'obstacles: a) obstacle rectangulaire,

b) obstacle rectangulaire arrondi.

De telles recherches sont nécessaires pour l'évaluation de la micro-échelle des modèles météorologiques développés pour prévoir la dispersion de polluant dans des secteurs urbains. Des émissions urbaines se produisent principalement dans une zone, là où l'écoulement atmosphérique est fortement troublé (zones de recirculation) par des 
bâtiments et d'autres obstacles [6]. Ces modèles sont utilisés dans des études d'incidence sur l'environnement. Ceci constitue un souci pour les décideurs dans des projets dont les décisions, basées sur les prévisions de tels modèles, peuvent avoir des conséquences économiques graves [7]. L'objectif de ce travail est de diminuer les dimensions des longueurs de réattachement et par suite les zones de recirculation.

Pour montrer l'influence de l'arrondissement sur la longueur de réattachement $X_{R} / h$ on étudie la variation de la longueur de réattachement en fonction du nombre de Reynolds $R e_{h}$ en prenant ce dernier égal à $R e_{h}=U_{g} h / v$ [8], et en fonction du rapport $h / H$ (hauteur de l'obstacle sur la hauteur du canal). Plusieurs travaux ont été menés pour étudier l'écoulement autour d'un obstacle rectangulaire; Castro [9] s'intéresse à l'influence de la longueur de réattachement sur la couche limite laminaire, il montre que pour $0.34<\delta / h<0.80$, la longueur de réattachement diminue en même temps que $\delta / h, \delta$ étant l'épaisseur de la couche limite laminaire.

Ce type d'étude est intéressant pour modéliser l'interaction des structures se trouvant dans la couche limite atmosphérique [2,10]. Dans une autre étude Bergles et Athanassiadis [11] étudient la longueur de réattachement $X_{R} / h$ en fonction du rapport décroissant de $I / h$ (longueur sur hauteur de l'obstacle) pour le cas d'un obstacle se trouvant dans une couche limite. laccarino ef Durbin [12] s'intéressent à $X_{R} / h$ et à la deuxième zone de recirculation dans le cas du régime non permanent ef le régime permanent.

Ils comparent plusieurs résultats s'appuyant sur l'expérimentation et d'autres s'appuyant sur des modèles de turbulence tels que L.E.S (Large Eddy Simulation) et R.A.N.S (Reynolds Averaged Navier-stokes equations) [12]. Martinuzzi et Tropea [13] s'intéressent à $X_{R} / h$ dans le cas d'un écoulement tridimensionnel autour d'obstacles, ils prennent en considération deux paramètres adimensionnels : en plus de $h / H$, ils étudient l'influence de $W / h$ (largeur sur hauteur de l'obstacle) et cela en utilisant des techniques de visualisation. Tropea et Gackstatter [8] mènent une comparaison entre un obstacle rectangulaire et un obstacle rectangulaire mince et cela pour trois valeurs de la hauteur de l'obstacle sur la hauteur du canal $h / H$. Ils arrivent à montrer que s'il n'y a pas naissance de la deuxième zone de recirculation (cas de $h / H=0.25$ ), la longueur de réattachement $X_{R} / h$ de l'obstacle rectangulaire mince est nettement supérieure à celle de l'obstacle rectangulaire. Pour un rapport de $h / H$ supérieur, cette constatation est inversée.

\section{Equations gouvernantes}

Les équations dynamiques des écoulements permanents à propriétés physiques constantes s'écrivent : 
- Equation du mouvement :

$\rho \frac{d \vec{V}}{d t}=\rho \vec{F}-\operatorname{gra} \vec{d} p+\frac{1}{3}(\mu \operatorname{gra} \vec{d} \operatorname{div} \vec{V})+\mu \Delta \vec{V}$

où $\rho$ représente la masse volumique du fluide, $\vec{V}$ le vecteur vitesse, $\vec{F}$ les forces de surfaces, $p$ la pression, $\mu$ la viscosité dynamique du fluide.

- Equation de continuité :

$\frac{\partial \rho}{\partial t}+\operatorname{div} \rho \vec{V}=0$

L'écoulement est supposé entre deux plaques planes parallèles avec existence d'obstacles. Les équations précédentes deviennent comme suit :

- Equation du mouvement suivant la direction $x$ et $y$ respectivement :

$$
\begin{aligned}
& \frac{\partial(\mathrm{uu})}{\partial \mathrm{x}}+\frac{\partial(\mathrm{uv})}{\partial \mathrm{y}}=\frac{\partial}{\partial \mathrm{x}}\left(\mathrm{v} \frac{\partial \mathrm{u}}{\partial \mathrm{x}}\right)-\frac{\partial \mathrm{p}}{\partial \mathrm{x}} \\
& \frac{\partial(\mathrm{uv})}{\partial \mathrm{x}}+\frac{\partial(\mathrm{vv})}{\partial \mathrm{y}}=\frac{\partial}{\partial \mathrm{y}}\left(v \frac{\partial \mathrm{v}}{\partial \mathrm{y}}\right)-\frac{\partial \mathrm{p}}{\partial \mathrm{y}}
\end{aligned}
$$

- Equation de continuité :

$$
\frac{\partial \mathbf{u}}{\partial \mathbf{x}}+\frac{\partial \mathbf{v}}{\partial \mathbf{y}}=\mathbf{O}
$$

La résolution de ces équations nous permet de tracer les lignes de courant et la distribution du champ des vitesses autour de l'obstacle. La solution analytique est donnée pour des cas très simples, et pour des cas complexes une approche numérique est inévitable.

\section{Partie expérimentale}

Les expériences sont effectuées dans un canal en plexiglas ayant une veine d'essai de $0.6 \mathrm{~cm} \times 16 \mathrm{~cm}$. Le lieu, où on place les obstacles, se trouve à une distance $\mathrm{de} 70 \mathrm{~cm}$ loin de l'entrée du canal. L'arrondissement existant à l'arête aval de l'obstacle a un rayon de courbure qui vaut 0.2 fois la hauteur de l'obstacle. L'air qui alimente le canal est mélangé avec des gouttelettes d'eau pulvérisées servant comme traceurs. Une taille de $0.3 \mu \mathrm{m}$ de ces traceurs donne un signal Doppler de bonne qualité représentatif de la vitesse instantanée des particules fluides. La mesure de la vitesse est obtenue par l'anémomètre Laser à effet Doppler suivant la direction principale de l'écoulement. Le système optique est constitué d'une source laser de type He-Ne de $10 \mathrm{~mW}$ de puissance 
et de polarisation linéaire. Le photodétecteur de type APD du système LDA est relié à un ordinateur au moyen d'une carte d'acquisition de type DSP. Un filtre passe-bande placé entre le photodétecteur et l'ordinateur nous permet de filtrer le signal acquis et donc de supprimer le bruit et obtenir un signal Doppler aussi meilleur que possible à l'aide de deux filtres de type passe-bas et passe-haut. Le filtre passe-bande a une sensibilité qui varie de $5 \mathrm{kHz}$ à $10 \mathrm{MHz}$. La montée en hauteur du dispositif de mesure est réalisée grâce à un système élévateur qui peut varier de $0.01 \mathrm{~mm}$. Une schématisation du problème étudié est donnée à la Figure 2.

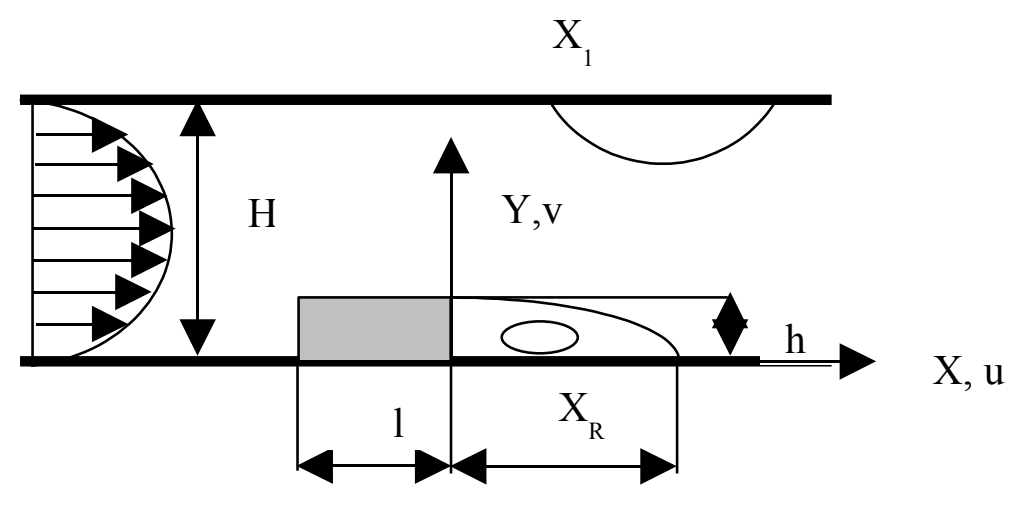

Figure 2 : Schématisation de l'écoulement autour d'un obstacle

\section{Résultats et discussion}

\section{3-1. Profils de vitesses}

La compréhension de l'effet de chaque type d'obstacle étudié peut être quantifiée en traçant les profils des vitesses en trois endroits du canal : en amont, sur et en aval de l'obstacle. La première section se trouve loin de l'entrée du canal où l'écoulement est considéré comme établi, ceci correspond à une distance supérieure à $70 \mathrm{~cm}$ loin de l'entrée. Dans cette section (section amont de l'obstacle), le profil de vitesse est parfaitement parabolique (Figure 3), obstacle rectangulaire et (Figure 4), obstacle rectangulaire a bout arrondi), dans cet endroit l'écoulement n'est pas perturbé par la présence de l'obstacle. 


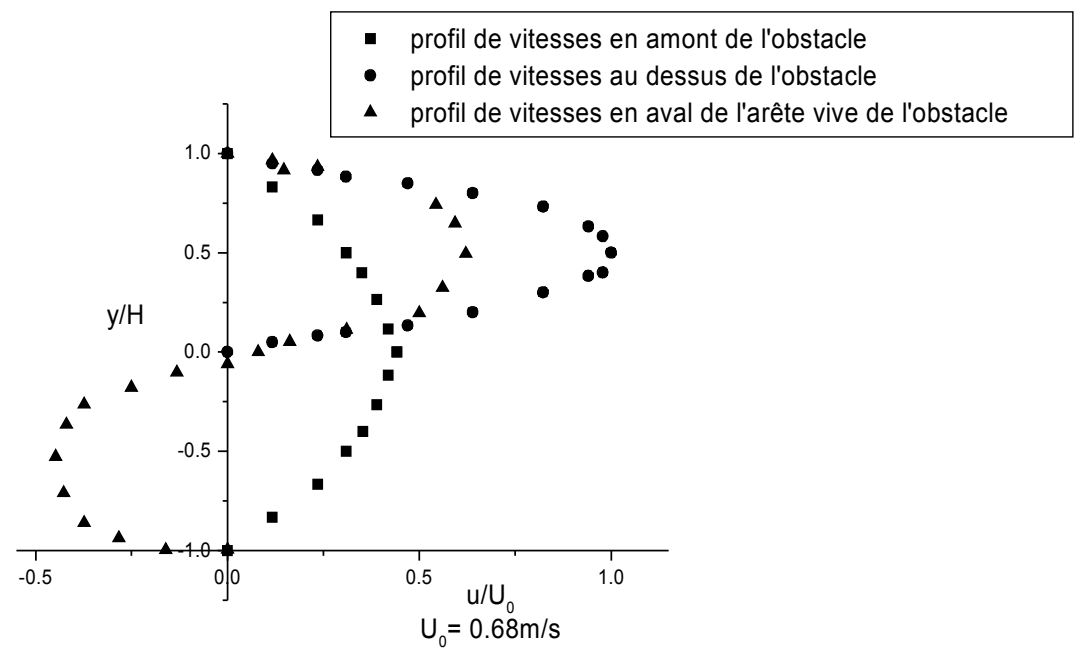

Figure 3 : Profil de vitesses pour l'obstacle rectangulaire, $\frac{h}{H}=0.5$ (incertitude pour $y: \pm 0.003 \mathrm{~mm}$ et $\mathrm{u}: \pm 4 \%$ )

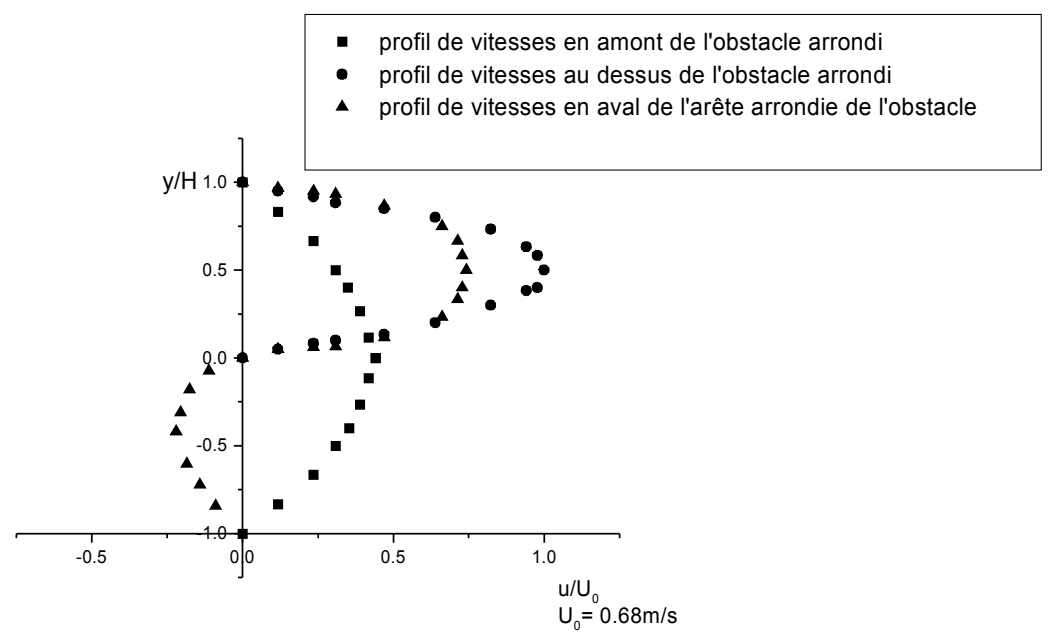

Figure 4 : Profil de vitesses pour l'obstacle rectangulaire arrondi, $\frac{h}{H}=0.5$ (incertitude pour $y: \pm 0.003 \mathrm{~mm}$ et $\mathrm{u}: \pm 4 \%$ )

La deuxième section se trouve juste au-dessus de l'obstacle, les profils de vitesses pour les deux types d'obstacles sont paraboliques comme le cas de la première section sauf 
que la vitesse en cet endroit est nettement supérieure qu'à l'entrée car l'écoulement est accéléré à cause du rétrécissement de la section du passage de l'écoulement; on ne distinguera donc pas de différence entre les deux types d'obstacles étudiés. En aval de l'obstacle (Figure 5), on peut quantifier deux grandes zones distinctes : une zone où les particules fluides suivent la direction principale de l'écoulement, dans cet endroit le profil de vitesses est encore parabolique mais ayant une envergure faible que celle de la deuxième section.

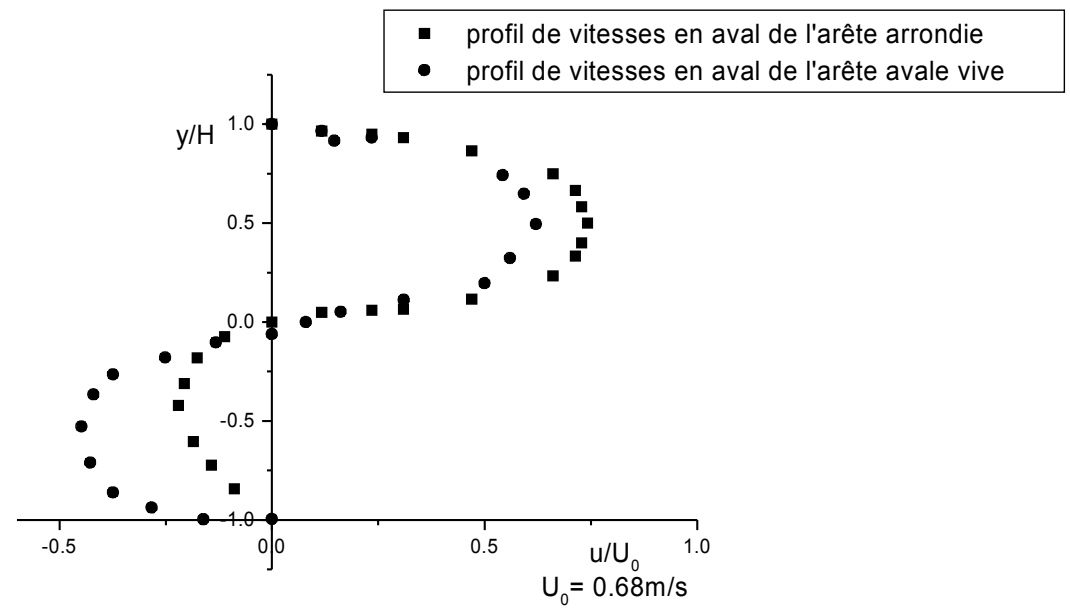

Figure 5 : Comparaison des profils de vitesses pour l'obstacle rectangulaire et rectangulaire arrondi, $\frac{h}{H}=0.5$ (incertitude pour $y: \pm 0.003 \mathrm{~mm}$ et $u: \pm 4 \%)$

La deuxième zone est celle où il existe la zone de recirculation, l'écoulement est inversé ce qui provoque le décollement de l'écoulement au niveau de la paroi inférieure du canal, les profils de vitesses sont dirigés dans le sens opposé de l'écoulement. L'obstacle rectangulaire à bout arrondi possède un profil de vitesse dont les dimensions sont très inférieures à celles de l'obstacle rectangulaire à arête aval vive. Cela se répercute sur les longueurs de réattachement et les dimensions des zones de recirculation des deux modèles d'obstacles à étudier.

\section{3-2. Zones et longueurs de réattachement}

Les résultats de la longueur de réattachement de l'obstacle rectangulaire et celle de l'obstacle rectangulaire avec arrondissement sont présentés dans les Figures 6 et 7 ; ces figures donnent la longueur de réattachement $X_{R} / h$ en fonction du nombre de Reynolds $R e_{h}$. Les résultats obtenus dans le cas de l'obstacle rectangulaire (Figure 6) 
sont comparés avec ceux de Tropea et Gackstatter [8], ces graphes montrent que ces résultats sont en bonne concordance.

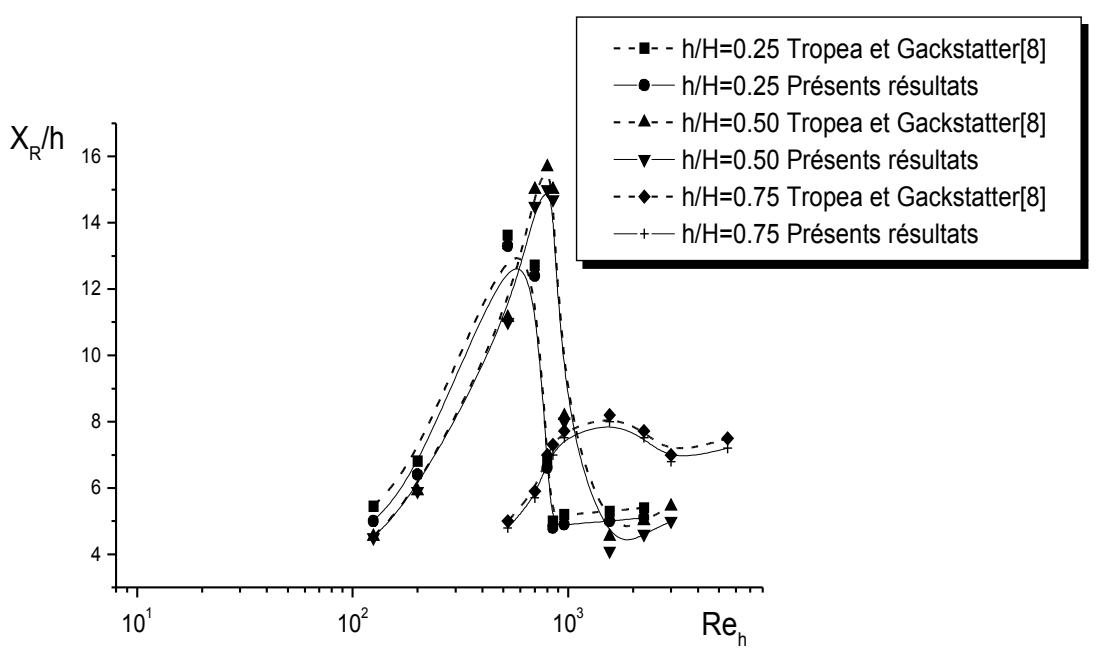

Figure 6 : La longueur de réattachement en fonction du nombre de Reynolds pour un obstacle rectangulaire (Incertitude pour $R_{h}: \pm 3 \%$ et $X_{R} / h: \pm 4.5 \%$ )

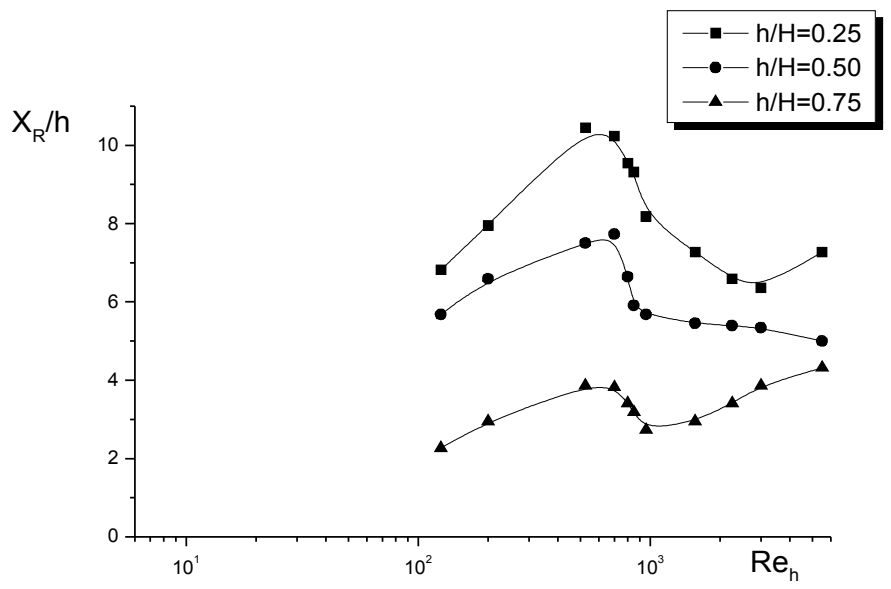

Figure 7 : La longueur de réattachement en fonction du nombre de Reynolds pour un obstacle rectangulaire arrondi (Incertitude pour $\operatorname{Re}_{h}: \pm 3 \%$ et $X_{R} / h: \pm 4.5 \%$ )

Ces graphes ont été construits pour trois régions : une région laminaire, où la longueur de réattachement $X_{R} / h$ augmente avec le nombre de Reynolds; une région de transition 
où une augmentation brusque de $X_{R} / h$ est remarquée et enfin une région turbulente où la longueur de réattachement commence à diminuer progressivement. Pour $h / H=0.25$, on constate que la longueur de réattachement $X_{R} / h$ de l'obstacle rectangulaire est nettement supérieure à celle de l'obstacle rectangulaire avec arrondissement comme il est indiqué dans la Figure 8, car il n'y a pas de naissance de la deuxième zone de recirculation pour le cas de l'obstacle rectangulaire, mais au contraire, cette zone prend naissance dans le cas de l'obstacle rectangulaire avec arrondissement (Figure 9).

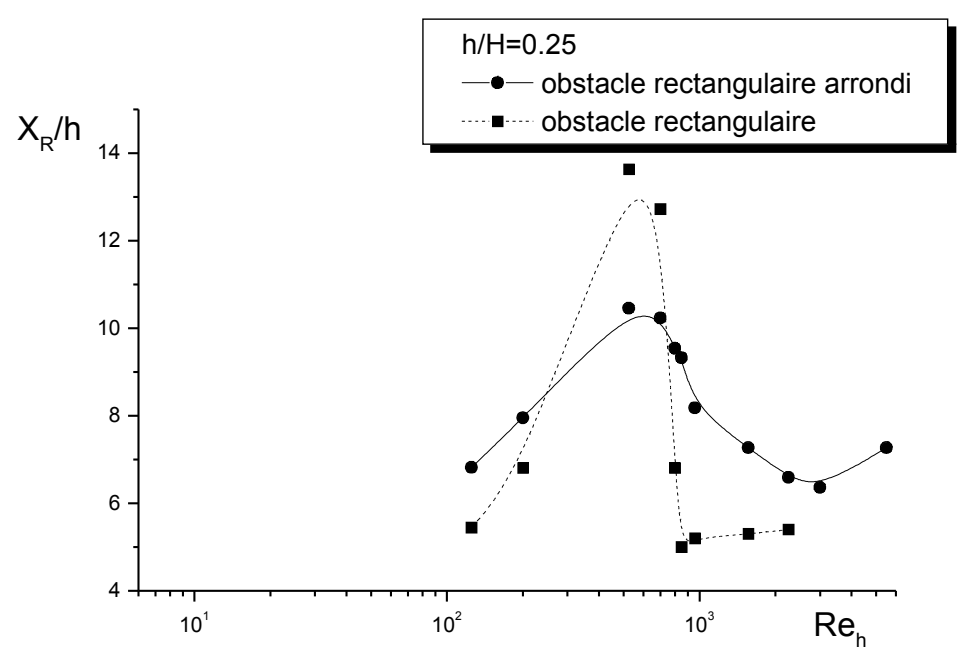

Figure 8 : Comparaison de la longueur de réattachement des obstacles rectangulaire et rectangulaire arrondi $\left(h / H=0.25\right.$ (incertitude pour $\mathrm{Re}_{h}: \pm 3 \%$ et $X_{R} / h: \pm 4.5 \%$ )

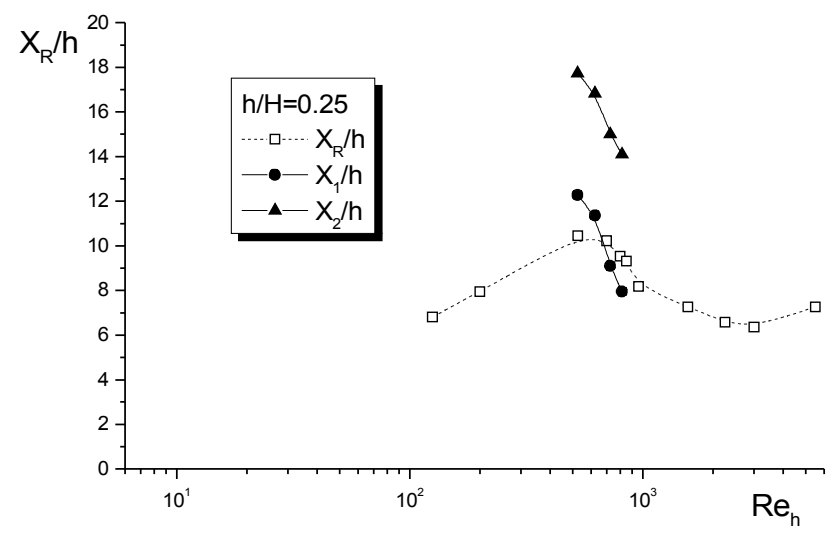

Figure 9 : La deuxième zone de recirculation pour un obstacle rectangulaire arrondi $\left(h / H=0.25\right.$ ) (incertitude pour $R_{h}: \pm 3 \%$ et $X_{R} / h: \pm 4.5 \%$ ) 
Pour un rapport de $h / H=0.5$ la longueur de réattachement $X_{R} / h$ de l'obstacle rectangulaire est nettement supérieure à celle de l'obstacle rectangulaire avec arrondissement (Figure 10) qui a comme précédemment, une longueur de réattachement plus faible.

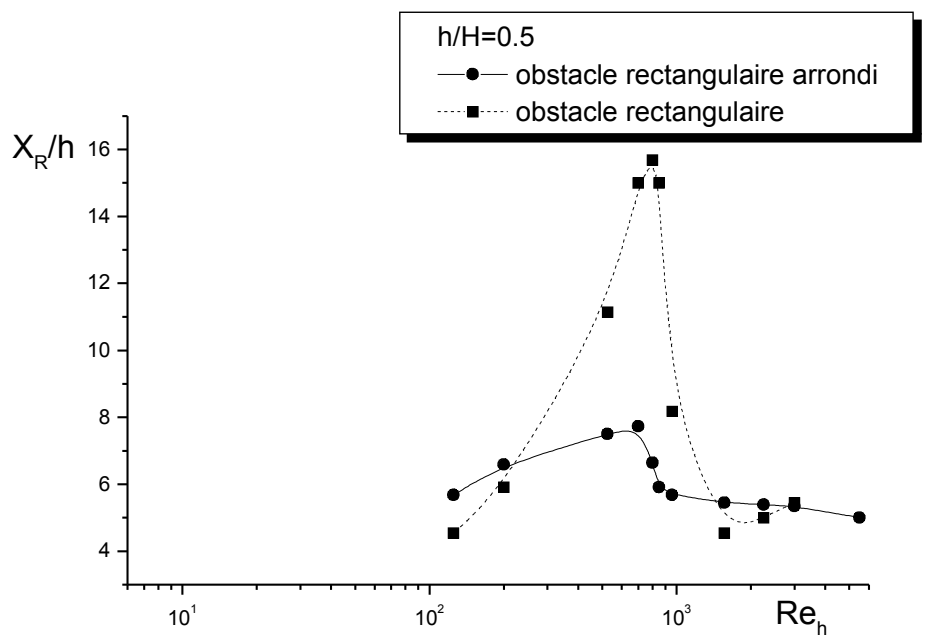

Figure 10 : Comparaison de la longueur de réattachement des obstacles rectangulaire et rectangulaire arrondi $(h / H=0.5)$ (incertitude pour $R e_{h}: \pm 3 \%$ et $X_{R} / h: \pm 4.5 \%$ )

Là aussi, il y a naissance de la deuxième zone de recirculation pour le cas de l'obstacle rectangulaire avec arrondissement (Figure II)

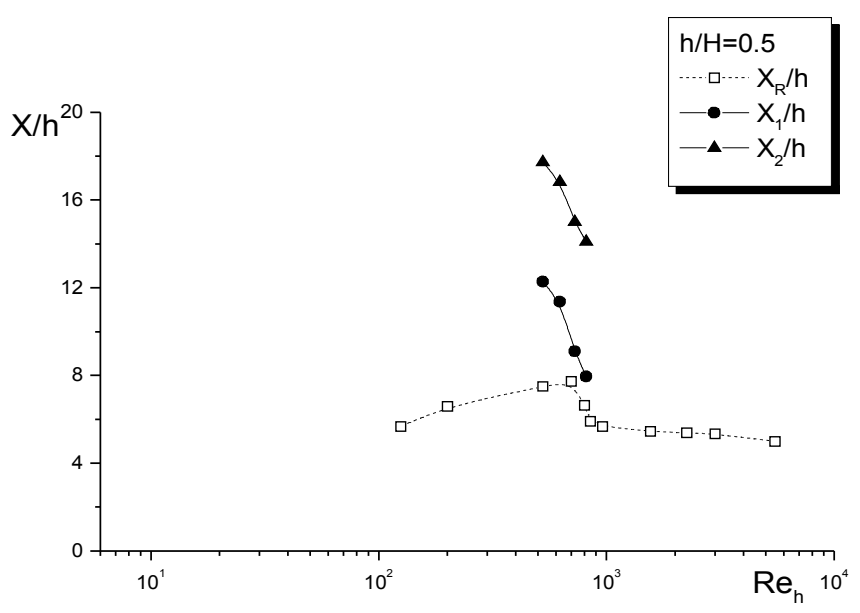

Figure 11 : La deuxième zone de recirculation pour un obstacle rectangulaire arrondi $(h / H=0.5)$ (incertitude pour $R e_{h}: \pm 3 \%$ et $X_{R} / h: \pm 4.5 \%$ ) 
Pour un rapport de $h / H=0.75$ la longueur de réattachement $X_{R} / h$ de l'obstacle rectangulaire est supérieure à celle de l'obstacle rectangulaire avec arrondissement (Figure 12).

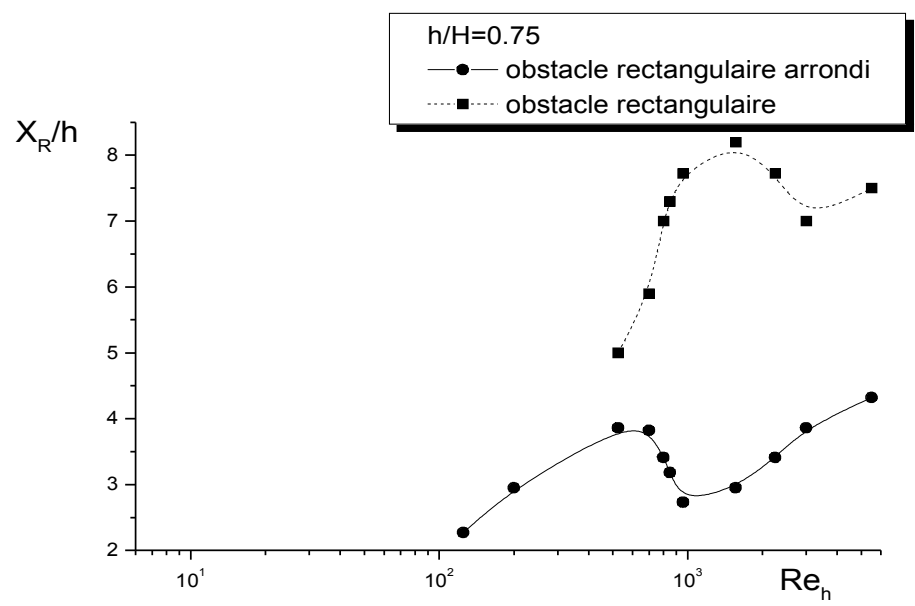

Figure 12 : Comparaison de la longueur de réattachement des obstacles rectangulaire et rectangulaire arrondi $(h / H=0.75)$ (incertitude pour $R_{h}: \pm 3 \%$ et $X_{R} / h: \pm 4.5 \%$ )

Cette fois il y a naissance de la deuxième zone de recirculation pour le cas de l'obstacle rectangulaire avec arrondissement et l'obstacle rectangulaire mais la deuxième zone de recirculation de l'obstacle rectangulaire avec arrondissement (Figure 13) est très importante que celle de l'obstacle rectangulaire (Figure 14),

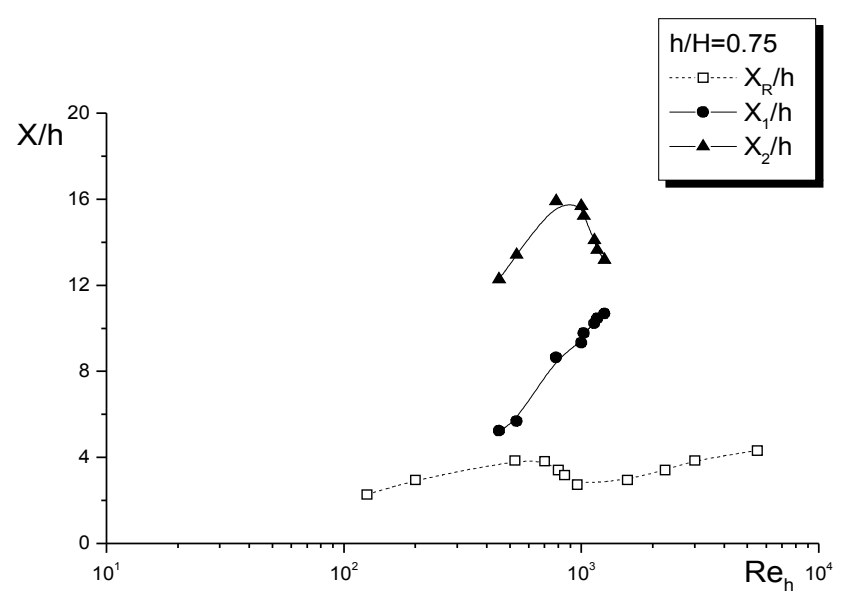

Figure 13 : La deuxième zone de recirculation pour un obstacle rectangulaire arrondi $(h / H=0.75)$ (incertitude pour $R_{h}: \pm 3 \%$ et $X_{R} / h: \pm 4.5 \%$ ) 


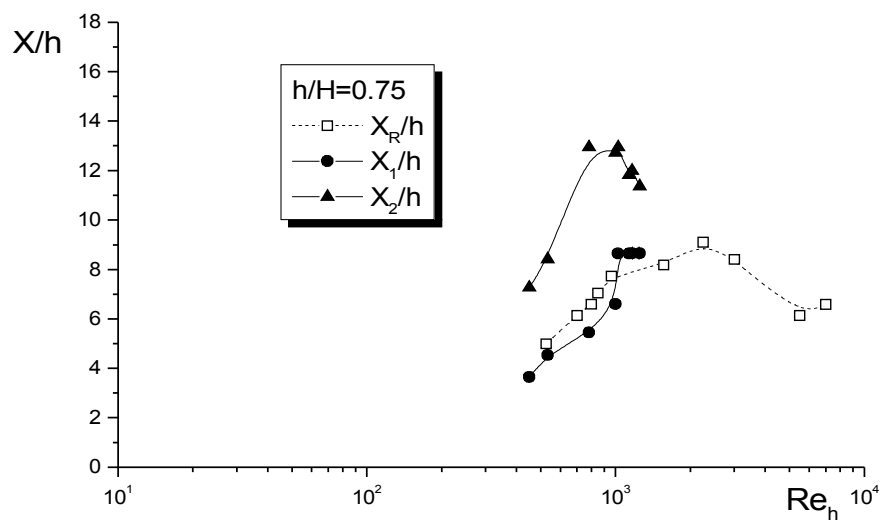

Figure 14 : La deuxième zone de recirculation pour un obstacle rectangulaire $(h / H=0.75)$ (incertitude pour $R e_{h}: \pm 3 \%$ et $X_{R} / h: \pm 4.5 \%$ )

car le rapport $h / H$ important dans le cas de l'obstacle rectangulaire arrondi crée une interaction entre l'effet de l'arrondissement représenté par une variation progressive de la pression en aval de l'obstacle, et la deuxième zone de recirculation puisque la distance entre la position de l'arrondissement de l'obstacle et la paroi supérieure du canal devient moins importante, ce qui permet d'augmenter les dimensions de la deuxième zone de recirculation, et par conséquent, la diminution de la longueur de réattachement $X_{R} / h$ de l'obstacle rectangulaire arrondi.

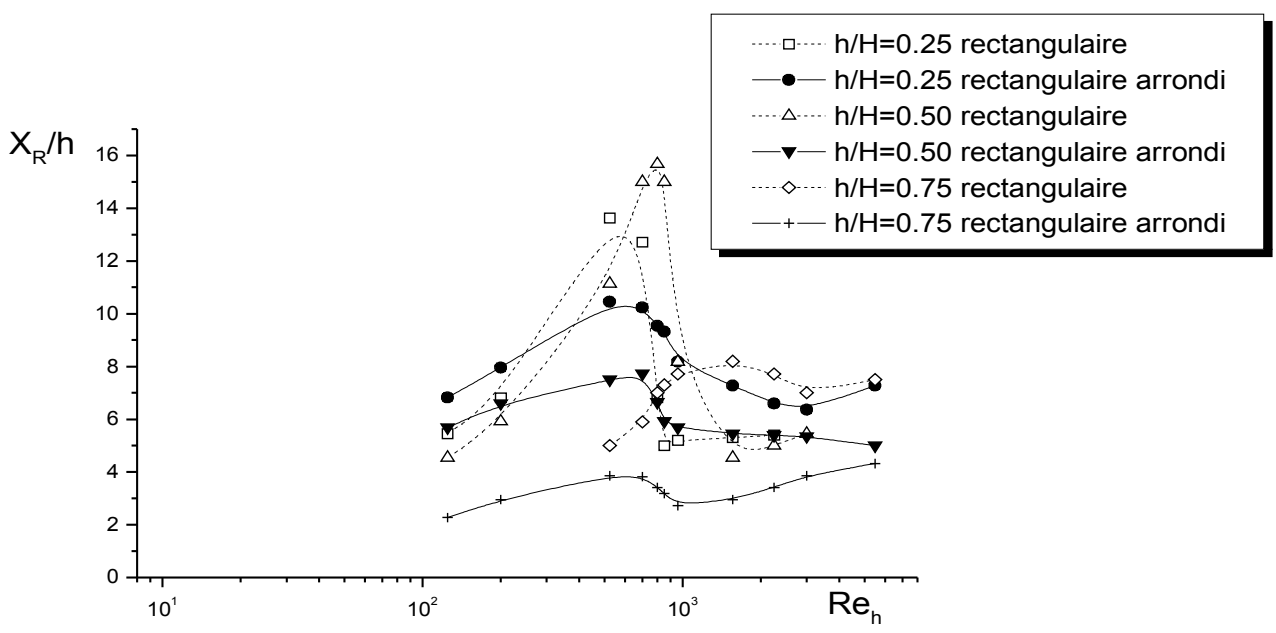

Figure 15 : Comparaison de la longueur de réattachement des obstacles rectangulaire et rectangulaire arrondi (incertitude pour $R_{h}: \pm 3 \%$ et $X_{R} / h: \pm 4.5 \%$ ) 
La Figure 15 donne l'évolution de la longueur de réattachement $X_{R} / h$ pour les trois rapports de $h / H_{\text {; }}$ cette figure montre l'effet de la forme arrondie se trouvant à la fin de l'obstacle sur la variation des dimensions de $X_{R} / h$. En effet, on constate que l'existence de la forme arrondie à la fin de l'obstacle rectangulaire diminue considérablement la longueur de réattachement pour les trois rapports de $h / H$ et que l'obstacle rectangulaire arrondi a des longueurs de réattachement plus faibles que celle de l'obstacle rectangulaire. On constate encore que $X_{R} / h$ diminue de plus en plus au fur et à mesure que le rapport $h / H$ augmente. Ceci peut être expliqué dans le cas de l'obstacle rectangulaire arrondi, de la façon suivante : le courant ne se sépare que pour une appréciable valeur de la composante transversale $v$ de la vitesse, et l'arrondissement existant à la fin de l'obstacle facilite l'écoulement suivant la direction principale de l'écoulement ce qui permet d'augmenter la composante $\boldsymbol{u}$ de la vitesse et de diminuer considérablement la longueur de réattachement.

\section{Conclusion}

L'écoulement bidimensionnel autour d'obstacles est étudié. L'influence de $h / H$ sur les longueurs de réattachement est discutée ; les résultats sont en bonne concordance avec ceux de Tropea et Gackstatter [8]. Un nouveau paramètre est introduit dans cette étude, c'est l'effet de la forme arrondie existant au bout de l'obstacle rectangulaire sur les longueurs de réattachement. Les résultats obtenus montrent que l'obstacle rectangulaire arrondi possède des longueurs de réattachement les plus faibles en comparant avec celles de l'obstacle rectangulaire et, cela quelque soit le rapport $h / H$, et que ces longueurs de réattachement (pour ce modèle d'obstacle) décroissent considérablement au fur et à mesure que le rapport $h / H$ devient important. Si l'obstacle rectangulaire crée un changement brusque de pression en aval de l'obstacle [14], I'obstacle rectangulaire à bout arrondi crée une variation progressive en aval de ce modèle d'obstacle, ce qui permet de diminuer considérablement les dimensions des longueurs de réattachement.

\section{Références}

[1]- K. Aliane , 0. Sebbane, A. Hadjoui , "Etude dynamique des modèles de refroidissement des aubes des turbomachines", Proceedings du $11^{\text {èmes }}$ journées Internationales de Thermique, Alger (Algerie), 21-23 Juin 2003, (2003) 315-320.

[2] - A. Hadjoui, 0. Sebbane, K. Aliane, A. Azzi, "Etude de l'apparition des zones tourbillonnaires au sein d'un écoulement confronté à des obstacles se trouvant d̀ l'entrée d'un canal', proceedings du $9^{\text {ème }}$ Congrès de la Société Française de Génie des Procédés, Saint-Nazaire, (France), 9-11 septembre 2003, (2003) 224-229. 
[3] - G. Bitsuamlak, T. Stathopoulos, C. Bedard, "Effects of upstream two-dimensional hills on design wind loads: a computational approach. ", Wind and Structures, 9 (1) (2006) 37-58.

[4] - O. Sebbane, A. Hadjoui, K. Aliane, A. Azzi, "Nouvelle méthode de visualisation des écoulement à très grand nombre de Reynolds", proceedings du $9^{\text {ème }}$ Congrès de la Société Française de Génie des Procédés, Saint-Nazaire, (France), 9-11 septembre 2003, (2003). 259-264.

[5] - K. Aliane, 0. Sebbane, A. Hadjoui, A. Azzi, R. Saim, "Contribution à l'étude de l'influence de la distance inter- obstacle sur la génération des zones tourbillonnaires au sein d'un écoulemenf', Proceedings $\mathrm{du} \mathrm{I}^{\text {er }}$ Congrès International de Mécanique, Constantine, Algérie, 14-16 Décembre 2002, (2002), 500-507.

[6] - B. Blocken, J. Carmeliet, "The influence of the wind blocking effect by a building on its wind-driven rain exposure. "Journal of Wind Engineering and Industrial Aerodynamics, 94 (2) (2006) 101-127.

[7] - A. K. M. Chua, R.C.W. Kwokb, K.N. Yua, "Study of pollution dispersion in urban areas using Computational Fluid Dynamics (CFD) and Geographic Information System (GIS) ", Environmental Modeling \& Software, 20 (2005) 273-277.

[8] - C. D. Tropea, R. Gackstatter, "The flow over two -dimensional surface-mounted obstacles at low Reynolds numbers", ASME Journal of Fluids Engineering. vol. 107 (1985) 489-494.

[9] - I. P. Castro, "Measurements in shear layers separating from surface-mounted bluff bodies", J. Wind Eng. and Ind. Aero., vol. 7, (1981) 253-257.

[10] - S. Dupont, "Etude numérique de la couche de surface atmosphérique au-dessus de rugosités du sol dans une configuration en damier", thèse de doctorat, Ecole Centrale de Nantes, (1997).

[11] - G. Bergeles, N. Athanassiadis, "The flow past a surface-mounted obstacles", ASME Journal of Fluids Engineering. vol. 105 (1983) 461-467.

[12] - G. laccarino, P. Durbin, "Unsteady 3D RANS simulations using the $v$ 2-f, model", Center for Turbulence Research, Annual Research Briefs, (2000) 263-269.

[13] - R. Martinuzzi, C. Tropea, "The flow around surface-mounted prismatic obstacles placed in a fully developed channel flow", ASME Journal of Fluids Engineering. vol. 115 (1993) pp. 85-92.

[14] - H. J. Hussein, R.J. Martinuzzi, "Energy Balance for the Turbulent Flow Around a Surface Mounted Cube Placed in a Channel", Phys. Fluids. Vol 8, (1996) 764-780. 